\title{
Direct Beamformer Estimation for Hybrid Architecture in mmWave Dynamic TDD System
}

\author{
Prashant Kumar Shah*, Krishna Joshi*, Satya Joshi*, Antti Tolli*, Kenta Umebayashi ${ }^{\dagger}$ \\ ${ }^{*}$ Centre for Wireless Communications, University of Oulu, P.O. Box 4500, 90014, Finland \\ ${ }^{\dagger}$ Dept. of Electrical and Electronic Engineering, Tokyo University of Agriculture and Technology, Japan \\ *\{prashant.shah, krishna.joshi, satya.joshi, antti.tolli\}@oulu.fi, † ume_k@cc.tuat.ac.jp
}

\begin{abstract}
The problem of weighted sum-rate maximization for hybrid architecture in millimeter wave communications with dynamic time division duplexing is considered. We propose an over the air pilot-aided direct beamforming estimation technique for the considered problem, by exploiting the channel reciprocity. Proper selection of analog precoder/combiner not only suppresses large part of interference, but also ensures that less coordination is required for digital precoder/combiner design. We design analog precoder/combiner for each data stream in such a way that its signal strength is maximized; this strategy also minimizes large part of inter-stream interference that may arise due to the main lobe of analog beamformer. Then we design digital beamformer such that inter-stream interference is further mitigated, maximizing the overall weighted sum-rate of the network.
\end{abstract}

\section{INTRODUCTION}

Due to proliferation of smart wireless devices the demand for wireless services with asymmetric uplink (UL) and downlink (DL) traffic is ever increasing. In traditional wireless networks with frequency division duplexing (FDD), the UL and DL capacity is determined by fixed frequency allocation [1]. Thus, FDD based systems often lead to poor spectrum utilization in case of asymmetric UL/DL traffic. In time division duplexing (TDD), it is possible to change the UL and DL capacity ratio dynamically according to the need, improving overall spectrum utilization of the network [2], [3]. Thus, dynamic TDD is becoming an essential feature for the emerging wireless communication standards [4].

In dynamic TDD systems, a same time-slot can be used for UL/DL transmissions in adjacent cells; unfortunately which also induces complicated interference scenarios [2], [3]. That is, in addition to DL-to-DL and UL-to-UL interference, there appear additional two different types of cross-cell interferences (i.e., DL-to-UL and UL-to-DL interference). Because of these four different types of interference scenarios, interference management is more challenging in dynamic TDD systems [5].

The use of multiple-input multiple-output (MIMO) techniques have been widely studied in order to manage the complicated interference scenarios in the dynamic TDD systems, e.g., [6]-[10]. The MIMO techniques can provide higher spectral efficiency and enhance the reliability of

This work was supported by the European Commission in the framework of the H2020-EUJ-02-2018 project 5G-Enhance (Grant agreement no. 815056), the Academy of Finland 6Genesis Flagship (grant no. 318927), and the Ministry of Internal Affairs and Communications (MIC) of Japan. wireless links, when the CSI is known [11]. By exploiting channel reciprocity, the direct beamformer estimation strategies for dynamic TDD have been provided in [6], [8]. Recently due to the scarcity of radio spectrum in the sub-6 GHz spectrum band, there has been growing interest in the exploration of underutilized millimeter wave (mmWave) frequency spectrum [12]. The use of dynamic TDD in mmWave band can further increase the capacity of communication networks. Unfortunately, the algorithms that have been proposed in the literature, e.g., [6]-[10] for dynamic TDD systems are not suitable for mmWave large scale MIMO systems.

The use of fully-digital beamforming for mmWave large scale MIMO systems is not practically feasible, due to its huge implementation cost and power consumption [13][15]. In the hybrid architecture less number of radio frequency (RF) chains can be deployed compared with the number of antennas, minimizing the implementation cost and power consumption. Thus, the use of hybrid architecture (analog/digital beamforming) is a potential solution for mmWave communications [13], [14]. However, the design of hybrid analog/digital beamforming for mmWave communications in the dynamic TDD systems is challenging.

We consider the problem of weighted sum-rate maximization (WSRMax) for mmWave communications with dynamic TDD. The main contribution of the paper is to propose a direct beamformer estimation approach for hybrid architecture in mmWave frequency band with dynamic TDD. In large scale MIMO system CSI acquisition is substantially challenging and resource consuming. Hence, we propose an over-the-air (OTA) pilot-aided beamforming technique by utilizing effective channel [16]. We first design analog precoder/combiner for each data stream in such a way that its signal strength is maximized, ignoring the inter-stream interference. This strategy, in mmWave communications with large number of antennas, also minimizes the inter-stream interference that may cause by the main lobe of analog beamformer. Then we design digital beamformer such that inter-stream interference is further mitigated, maximizing the overall weighted sum-rate of the network. It is worth noting that for the design of digital beamformer, somewhat similar techniques were used in [8] in the context of fully-digital MIMO architecture. However, the problem addressed in this paper is substantially more challenging due to the need of 
hybrid architecture for mmWave communications.

\section{SYSTEM MODEL}

We consider a multi-cell multi-user MIMO system operating in dynamic TDD mode with $B$ base stations (BSs) each equipped with $N$ antennas. The set of BSs is denoted by $\mathcal{B}$ and we label them with the integer values $b=1,2, \ldots, B$. We denote the set of BSs in DL mode by $\mathcal{B}_{\mathrm{DL}}$ and the set of BSs in UL mode by $\mathcal{B}_{\mathrm{UL}}$. Hence, we have $\mathcal{B}=\mathcal{B}_{\mathrm{DL}} \cup \mathcal{B}_{\mathrm{UL}}$. We denote the set of users served by the BS $b$ by $\mathcal{K}_{b}$, and we label them with the integer values $k=1,2, \ldots, K_{b}$. Each user is equipped with $M$ receive antennas. We assume that the BSs are equipped with large number of antenna elements compared with users, i.e., $N>>M$, and hence we assume that each BS has hybrid precoding/decoding structure with $N^{\mathrm{RF}} \mathrm{RF}$ chains (i.e., analog/digital precoding and combining is performed at BSs) ${ }^{1}$. It is assumed that for each user $d$ data streams are allocated, with $d \leq \min (N, M)$.

\section{A. Downlink Scenario}

Let $\mathbf{U}_{b} \in \mathbb{C}^{N \times N^{\mathrm{RF}}}$ denote analog precoder of BS $b$ and $\mathbf{V}_{b k} \in \mathbb{C}^{N^{\mathrm{RF}} \times d}$ denote digital precoder associated with $k$ th user of BS $b$. Then the signal received by $k$ th user of BS $b$ can be expressed as

$$
\begin{aligned}
& \mathbf{y}_{b k}=\underbrace{\mathbf{H}_{b, b k} \mathbf{U}_{b} \mathbf{V}_{b k} \mathbf{s}_{b k}}_{\text {Desired Signal }}+\underbrace{\mathbf{H}_{b, b k} \mathbf{U}_{b} \sum_{\substack{l \in \mathcal{K}_{b} \\
l \neq k}} \mathbf{V}_{b l} \mathbf{s}_{b l}}_{\text {Intra-cell DL-to-DL Interference }} \\
& +\underbrace{\sum_{\substack{p \in \mathcal{B}_{\mathrm{DL}} \\
p \neq b}} \mathbf{H}_{p, b k} \mathbf{U}_{p} \sum_{l \in \mathcal{K}_{p}} \mathbf{V}_{p l} \mathbf{s}_{p l}}_{\text {Inter-cell DL-to-DL Interference }}+\underbrace{\sum_{n \in \mathcal{B}_{\mathrm{UL}}} \sum_{l \in \mathcal{K}_{p}} \overline{\mathbf{H}}_{n l, b k} \overline{\mathbf{V}}_{n l} \mathbf{r}_{n l}}_{\text {UL-to-DL Interference }} \\
& +\underbrace{\mathbf{z}_{b k}}_{\text {noise }}
\end{aligned}
$$

where $\mathbf{H}_{b, b k} \in \mathbb{C}^{M \times N}$ is the channel matrix between $b$ th BS and $k$ th user of BS $b, \overline{\mathbf{H}}_{n l, b k} \in \mathbb{C}^{M \times M}$ is the channel matrix between $l$ th user of UL BS $n$ and $k$ th user of DL BS $b$, and $\mathbf{z}_{b k}$ is complex Gaussian noise with covariance $\sigma^{2} \mathbf{I}$. In expression (1), notation $\mathbf{s}_{b k} \in \mathbb{C}^{d \times 1}$ denotes information symbol vector associated with $k$ th user of BS $b$ such that $\mathbb{E}\left[\mathbf{s}_{b k} \mathbf{s}_{b k}^{\mathrm{H}}\right]=\mathbf{I}$. Furthermore, we assume that each element of analog precoder $\mathbf{U}_{b}$ is implemented using a phase shifter, such that $\left|\mathbf{U}_{b}(i, j)\right|^{2}=1$ for all $b \in \mathcal{B}_{\mathrm{DL}}$.

We assume a linear receiver, where information symbol is estimated by linearly combining the received signal vector $\mathbf{y}_{b k}$ with the combiner $\mathbf{W}_{b k} \in \mathbb{C}^{M \times d}$. Thus, the symbol $s_{b k}$ can be estimated as

$$
\hat{\mathbf{s}}_{b k}=\mathbf{W}_{b k}^{\mathrm{H}} \mathbf{y}_{b k} .
$$

The mean-squared-error (MSE) matrix associated with $k$ th user of DL BS $b$ can be expressed as

$$
\mathbf{E}_{b k}^{\mathrm{DL}}=\mathbb{E}\left[\left(\mathbf{s}_{b k}-\hat{\mathbf{s}}_{b k}\right)\left(\mathbf{s}_{b k}-\hat{\mathbf{s}}_{b k}\right)^{\mathrm{H}}\right]
$$

\footnotetext{
${ }^{1}$ For simplicity, we assume that BS has hybrid architecture and user has fully-digital. But the method we propose can be easily extended for the case where both BSs and users have hybrid structure.
}

$$
\begin{aligned}
& =\mathbf{I}-\mathbf{V}_{b k}^{\mathrm{H}} \mathbf{U}_{b}^{\mathrm{H}} \mathbf{H}_{b, b k}^{\mathrm{H}} \mathbf{W}_{b k}-\left(\mathbf{V}_{b k}^{\mathrm{H}} \mathbf{U}_{b}^{\mathrm{H}} \mathbf{H}_{b, b k}^{\mathrm{H}} \mathbf{W}_{b k}\right)^{\mathrm{H}} \\
& +\mathbf{W}_{b k}^{\mathrm{H}} \mathbf{C}_{b k} \mathbf{W}_{b k},
\end{aligned}
$$

where $\mathbf{C}_{b k}$ represents the received signal covariance matrix and is expressed as

$$
\begin{aligned}
\mathbf{C}_{b k}= & {\left[\mathbf{H}_{b, b k} \mathbf{U}_{b}\left(\sum_{l \in \mathcal{K}_{b}} \mathbf{V}_{b l} \mathbf{V}_{b l}^{\mathrm{H}}\right) \mathbf{U}_{b}^{\mathrm{H}} \mathbf{H}_{b, b k}^{\mathrm{H}}\right.} \\
& +\sum_{\substack{p \in \mathcal{B}_{\text {DL }} \\
p \neq b}} \mathbf{H}_{p, b k} \mathbf{U}_{p}\left(\sum_{l \in \mathcal{K}_{p}} \mathbf{V}_{p l} \mathbf{V}_{p l}^{\mathrm{H}}\right) \mathbf{U}_{p}^{\mathrm{H}} \mathbf{H}_{p, b k}^{\mathrm{H}} \\
& \left.\quad+\sum_{n \in \mathcal{B}_{\mathrm{UL}}} \sum_{l \in \mathcal{K}_{p}} \overline{\mathbf{H}}_{n l, b k} \overline{\mathbf{V}}_{n l} \overline{\mathbf{V}}_{n l}^{\mathrm{H}} \overline{\mathbf{H}}_{n l, b k}^{\mathrm{H}}+\sigma^{2} \mathbf{I}\right] .
\end{aligned}
$$

We obtain the linear minimum mean-square error (LMMSE) receiver associated with $k$ th user of DL BS $b$ by minimizing (3), for fixed $\mathbf{V}_{b k}$, and it can be expressed as

$$
\mathbf{W}_{b k}=\mathbf{C}_{b k}^{-1}\left(\mathbf{H}_{b, b k} \mathbf{U}_{b} \mathbf{V}_{b k}\right) \text {. }
$$

When LMMSE receiver is used, MSE matrix (3) reduces to

$$
\tilde{\mathbf{E}}_{b k}^{\mathrm{DL}}=\mathbf{I}-\mathbf{V}_{b k}^{\mathrm{H}} \mathbf{U}_{b}^{\mathrm{H}} \mathbf{H}_{b, b k}^{\mathrm{H}} \mathbf{W}_{b k} .
$$

In this paper, we assume Gaussian signaling is used and interference from all other users and BSs are treated as noise. Thus, the rate of $k$ th user of DL BS $b$ can be expressed as

$R_{b k}=\log _{2}\left|\mathbf{I}+\mathbf{W}_{b k}^{\mathrm{H}} \mathbf{H}_{b, b k} \mathbf{U}_{b} \mathbf{V}_{b k} \mathbf{V}_{b k}^{\mathrm{H}} \mathbf{U}_{b}^{\mathrm{H}} \mathbf{H}_{b, b k}^{\mathrm{H}} \mathbf{W}_{b k} \mathbf{J}_{b k}^{-1}\right|$,

where $\mathbf{J}_{b k}$ is given by

$$
\begin{aligned}
\mathbf{J}_{b k}= & \mathbf{W}_{b k}^{\mathrm{H}}\left[\mathbf{H}_{b, b k} \mathbf{U}_{b}\left(\sum_{\substack{l \in \mathcal{K}_{b} \\
l \neq k}} \mathbf{V}_{b l} \mathbf{V}_{b l}^{\mathrm{H}}\right) \mathbf{U}_{b}^{\mathrm{H}} \mathbf{H}_{b, b k}^{\mathrm{H}}\right. \\
& +\sum_{\substack{p \in \mathcal{B}_{\mathrm{DL}} \\
p \neq b}} \mathbf{H}_{p, b k} \mathbf{U}_{p}\left(\sum_{l \in \mathcal{K}_{p}} \mathbf{V}_{p l} \mathbf{V}_{p l}^{\mathrm{H}}\right) \mathbf{U}_{p}^{\mathrm{H}} \mathbf{H}_{p, b k}^{\mathrm{H}} \\
& \left.+\sum_{n \in \mathcal{B}_{\mathrm{UL}}} \sum_{l \in \mathcal{K}_{p}} \overline{\mathbf{H}}_{n l, b k} \overline{\mathbf{V}}_{n l} \overline{\mathbf{V}}_{n l}^{\mathrm{H}} \overline{\mathbf{H}}_{n l, b k}^{\mathrm{H}}+\sigma^{2} \mathbf{I}\right] \mathbf{W}_{b k} .
\end{aligned}
$$

\section{B. Uplink Scenario}

The signal received by UL BS $n$ can be expressed as

$$
\begin{gathered}
\overline{\mathbf{y}}_{n}=\underbrace{\mathbf{H}_{n, n l}^{\mathrm{H}} \overline{\mathbf{V}}_{n l} \mathbf{r}_{n l}}_{\text {Desired Signal }}+\underbrace{\sum_{\text {Intra-cell UL-to-UL Interference }}^{\mathrm{H}} \overline{\mathbf{V}}_{n, n k} \mathbf{r}_{n k}}_{\substack{k \in \mathcal{K}_{n} \\
k \neq l}} \\
+\underbrace{\sum_{\substack{b \in \mathcal{B}_{\mathrm{UL}} \\
b \neq n}} \sum_{l \in \mathcal{K}_{b}} \mathbf{H}_{n, b l}^{\mathrm{H}} \overline{\mathbf{V}}_{b l} \mathbf{r}_{b l}}_{\text {Inter-cell UL-to-UL Interference }}+\underbrace{\sum_{b \in \mathcal{B}_{\mathrm{DL}}} \tilde{\mathbf{H}}_{b, n} \mathbf{U}_{b} \sum_{k \in \mathcal{K}_{b}} \mathbf{V}_{b k} \mathbf{s}_{b k}}_{\text {DL-to-UL Interference }} \\
+\underbrace{\overline{\mathbf{z}}_{n}}_{\text {noise }},
\end{gathered}
$$

where $\mathbf{H}_{n, n l}^{\mathrm{H}} \in \mathbb{C}^{N \times M}$ is the channel matrix between $n$th BS and $l$ th user of BS $n, \tilde{\mathbf{H}}_{b, n} \in \mathbb{C}^{N \times N}$ is the channel matrix between $b$ th DL BS and $n$th UL BS, $\overline{\mathbf{V}}_{n l} \in \mathbb{C}^{M \times d}$ denotes digital precoder and $\mathbf{r}_{n l} \in \mathbb{C}^{d \times 1}$ denotes information symbol associated with $l$ th user of UL BS $n$. We assume that $\mathbf{r}_{n l}$ is normalized such that $\mathbb{E}\left[\mathbf{r}_{n l} \mathbf{r}_{n l}^{\mathrm{H}}\right]=\mathbf{I}$. In expression (7), notation $\overline{\mathbf{z}}_{n}$ denotes a complex Gaussian noise with covariance $\sigma^{2} \mathbf{I}$.

We assume that each BS has hybrid precoder/combiner. Let $\overline{\mathbf{U}}_{n} \in \mathbb{C}^{N \times N^{\mathrm{RF}}}$ be an analog combiner of UL BS $n$ and 
$\overline{\mathbf{W}}_{n l} \in \mathbb{C}^{N^{\mathrm{RF}} \times d}$ be digital combiner associated with $l$ th user of UL BS $n$. Thus, the symbol $\mathbf{r}_{n l}$ can be estimated as

$$
\hat{\mathbf{r}}_{n l}=\mathbf{Q}_{n l}^{\mathrm{H}} \overline{\mathbf{y}}_{n}
$$

where $\mathbf{Q}_{n l}=\overline{\mathbf{U}}_{n} \overline{\mathbf{W}}_{n l}$. We assume that each element of analog combiner $\overline{\mathbf{U}}_{n}$ is implemented using a phase shifter, such that $\left|\overline{\mathbf{U}}_{n}(i, j)\right|^{2}=1$ for all $n \in \mathcal{B}_{\mathrm{UL}}$.

The MSE matrix associated with $l$ th user of UL BS $n$ can be expressed as

$$
\begin{aligned}
\mathbf{E}_{n l}^{\mathrm{UL}} & =\mathbb{E}\left[\left(\mathbf{r}_{n l}-\hat{\mathbf{r}}_{n l}\right)\left(\mathbf{r}_{n l}-\hat{\mathbf{r}}_{n l}\right)^{\mathrm{H}}\right] \\
& =\mathbf{I}-\overline{\mathbf{V}}_{n l}^{\mathrm{H}} \mathbf{H}_{n, n l} \mathbf{Q}_{n l}-\left(\overline{\mathbf{V}}_{n l}^{\mathrm{H}} \mathbf{H}_{n, n l} \mathbf{Q}_{n l}\right)^{\mathrm{H}}+\mathbf{Q}_{n l}^{\mathrm{H}} \overline{\mathbf{C}}_{n} \mathbf{Q}_{n l},
\end{aligned}
$$

where $\overline{\mathbf{C}}_{n}$ is given by

$$
\begin{aligned}
\overline{\mathbf{C}}_{n} & =\left[\sigma^{2} \mathbf{I}+\sum_{b \in \mathcal{B}_{\mathrm{DL}}} \tilde{\mathbf{H}}_{b, n} \mathbf{U}_{b}\left(\sum_{k \in \mathcal{K}_{b}} \mathbf{V}_{b k} \mathbf{V}_{b k}^{\mathrm{H}}\right) \mathbf{U}_{b}^{\mathrm{H}} \mathbf{H}_{b, n}^{\mathrm{H}}\right. \\
+ & \left.\sum_{k \in \mathcal{K}_{n}} \mathbf{H}_{n, n k}^{\mathrm{H}} \overline{\mathbf{V}}_{n k} \overline{\mathbf{V}}_{n k}^{\mathrm{H}} \mathbf{H}_{n, n k}+\sum_{\substack{b \in \mathcal{B}_{\mathrm{UL}} \\
b \neq n}} \sum_{l \in \mathcal{K}_{b}} \mathbf{H}_{n, b l}^{\mathrm{H}} \overline{\mathbf{V}}_{b l} \overline{\mathbf{V}}_{b l}^{\mathrm{H}} \mathbf{H}_{n, b l}\right] .
\end{aligned}
$$

The LMMSE receiver associated with $l$ th user of uplink BS $n$ can be obtained by minimizing (9) for fixed $\overline{\mathbf{V}}_{n l}$, and it can be expressed as

$$
\overline{\mathbf{W}}_{n l}=\left(\overline{\mathbf{U}}_{n}^{\mathrm{H}} \overline{\mathbf{C}}_{n} \overline{\mathbf{U}}_{n}\right)^{-1} \overline{\mathbf{U}}_{n}^{\mathrm{H}} \mathbf{H}_{n, n l}^{\mathrm{H}} \overline{\mathbf{V}}_{n l} .
$$

When LMMSE receiver is used, MSE matrix (9) reduces to

$$
\tilde{\mathbf{E}}_{n l}^{\mathrm{UL}}=\mathbf{I}-\overline{\mathbf{V}}_{n l}^{\mathrm{H}} \mathbf{H}_{n, n l} \overline{\mathbf{U}}_{n} \overline{\mathbf{W}}_{n l} \text {. }
$$

Then the rate of $l$ th user of UL BS $n$ can be expressed as

$$
\bar{R}_{n l}=\log _{2}\left|\mathbf{I}_{M}+\mathbf{Q}_{n l}^{\mathrm{H}} \mathbf{H}_{n, n l}^{\mathrm{H}} \overline{\mathbf{V}}_{n l} \overline{\mathbf{V}}_{n l}^{\mathrm{H}} \mathbf{H}_{n, n l} \mathbf{Q}_{n l} \overline{\mathbf{J}}_{n l}^{-1}\right|,
$$

where $\overline{\mathbf{J}}_{n l}$ is given by

$$
\begin{aligned}
& \overline{\mathbf{J}}_{n l}=\mathbf{Q}_{n l}^{\mathrm{H}}[ \sum_{\substack{k \in \mathcal{K}_{n} \\
k \neq l}} \mathbf{H}_{n, n k}^{\mathrm{H}} \overline{\mathbf{V}}_{n k} \overline{\mathbf{V}}_{n k}^{\mathrm{H}} \mathbf{H}_{n, n k} \\
&+\sum_{\substack{b \in \mathcal{B}_{\mathrm{UL}} \\
b \neq n}} \sum_{k \in \mathcal{K}_{b}} \mathbf{H}_{n, b k}^{\mathrm{H}} \overline{\mathbf{V}}_{b k} \overline{\mathbf{V}}_{b k}^{\mathrm{H}} \mathbf{H}_{n, b k} \\
&\left.+\sum_{b \in \mathcal{B}_{\mathrm{DL}}} \tilde{\mathbf{H}}_{b, n} \mathbf{U}_{b}\left(\sum_{k \in \mathcal{K}_{b}} \mathbf{V}_{b k} \mathbf{V}_{b k}^{\mathrm{H}}\right) \mathbf{U}_{b}^{\mathrm{H}} \tilde{\mathbf{H}}_{b, n}^{\mathrm{H}}+\sigma^{2} \mathbf{I}\right] \mathbf{Q}_{n l} .
\end{aligned}
$$

\section{PROBlem Formulation}

Let $\mu_{b k}$ and $\bar{\mu}_{n l}$ be arbitrary nonnegative weights assigned with $k$ th user of DL BS $b$ and $l$ th user of UL BS $n$, respectively. Assuming that the power allocation to $b$ th DL $\mathrm{BS}$ and $l$ th user of UL BS $n$ are subject to maximum power constraints $\sum_{k \in \mathcal{K}_{b}} \operatorname{Tr}\left(\mathbf{U}_{b} \mathbf{V}_{b k} \mathbf{V}_{b k}^{\mathrm{H}} \mathbf{U}_{b}^{\mathrm{H}}\right) \leq P_{b}^{\max }$ and $\operatorname{Tr}\left(\overline{\mathbf{V}}_{n l} \overline{\mathbf{V}}_{n l}^{\mathrm{H}}\right) \leq \bar{P}_{n l}^{\max }$, respectively, the problem of WSRMax with hybrid architecture at BSs can be expressed as

$$
\begin{array}{ll}
\text { maximize } & \sum_{b \in \mathcal{B}_{\mathrm{DL}}} \sum_{k \in \mathcal{K}_{b}} \mu_{b k} R_{b k}+\sum_{n \in \mathcal{B}_{\mathrm{UL}}} \sum_{l \in \mathcal{K}_{n}} \bar{\mu}_{n l} \bar{R}_{n l} \\
\text { subject to } & \sum_{k \in \mathcal{K}_{b}} \operatorname{Tr}\left(\mathbf{U}_{b} \mathbf{V}_{b k} \mathbf{V}_{b k}^{\mathrm{H}} \mathbf{U}_{b}^{\mathrm{H}}\right) \leq P_{b}^{\max }, \quad b \in \mathcal{B}_{\mathrm{DL}} \\
& \operatorname{Tr}\left(\overline{\mathbf{V}}_{n l} \overline{\mathbf{V}}_{n l}^{\mathrm{H}}\right) \leq \bar{P}_{n l}^{\max }, n \in \mathcal{B}_{\mathrm{UL}}, l \in \mathcal{K}_{\mathrm{n}} \\
& \left|\mathbf{U}_{b}(i, j)\right|^{2}=1 \quad \forall i, j, \quad b \in \mathcal{B}_{\mathrm{DL}} \\
& \left|\overline{\mathbf{U}}_{n}(i, j)\right|^{2}=1 \quad \forall i, j, \quad n \in \mathcal{B}_{\mathrm{UL}}
\end{array}
$$

with variables $\quad\left\{\mathbf{V}_{b k}, \mathbf{W}_{b k}, \mathbf{U}_{b}\right\}_{b \in \mathcal{B}_{\mathrm{DL}}, k \in \mathcal{K}_{\mathrm{b}}} \quad$ and $\left\{\overline{\mathbf{V}}_{n l}, \overline{\mathbf{W}}_{n l}, \overline{\mathbf{U}}_{n}\right\}_{n \in \mathcal{B}_{\mathrm{UL}}, l \in \mathcal{K}_{\mathrm{n}}}$. Expressions of $R_{b k}$ and $\bar{R}_{n l}$ are defined in (6) and (12), respectively.

\section{Algorithm DERIVATION}

In this section, we derive a fast but possibly suboptimal algorithm for NP-hard problem (13). We first design analog precoder/combiner for each data stream in such a way that its signal strength is maximized. Then digital precoder/combiner is designed such that inter-stream interference is further mitigated, maximizing problem (13).

\section{A. Analog Precoder/Combiner Design}

Various optimization techniques have been proposed to design analog precoder/combiner for the hybrid structure when the instantaneous CSI is known, e.g., [13], [17]. In this paper, we propose an OTA pilot-aided beamforming technique for problem (13) [16]. We use following two methods to design the analog precoder/combiner.

\section{1) User's position based analog precoder/combiner design}

In this method, we assign analog beamformer to the BSs from the predefined coodebook by exploiting the location of the user positions. Here, we briefly outline the procedure of selecting analog precoders for the DL BSs. Similarly, we can design analog combiners for the UL BSs.

Let $\mathcal{C}=\left\{\mathbf{c}_{1}\left(\alpha_{1}\right), \ldots, \mathbf{c}_{N}\left(\alpha_{N}\right)\right\} \in \mathbb{C}^{N \times N}$ be the analog codebook associated with a BS that satisfies constraints (13d) and (13e). For simplicity, we assume that beamformers $\left\{\mathbf{c}_{1}\left(\alpha_{i}\right)\right\}_{i=1, \ldots, N}$ are in two-dimensional space, and they are resolvable in angular domain (i.e., main lobe of $i$ th beam $\mathbf{c}_{i}\left(\alpha_{i}\right)$ is pointing toward angle $\alpha_{i}$ with respect to an antenna array; see Fig. 1). Then in two-dimensional space, the normal vector associated with the main lobe of $i$ th beam $\mathbf{c}_{i}\left(\alpha_{i}\right)$ can be expressed as $\hat{\mathbf{c}}_{i}=\left[\cos \left(\alpha_{i}\right) \sin \left(\alpha_{i}\right)\right]^{\mathrm{T}}$. Furthermore, let $\mathbf{x}_{b k}$ be the coordinate of $k$ th user associated with BS $b$, and its unit direction vector be $\hat{\mathbf{x}}_{b k}=\mathbf{x}_{b k} /\left\|\mathbf{x}_{b k}\right\|_{2}$. Then in the case of line-of-sight (LOS) scenario, the best analog beam that can be associated with $k$ th user of BS $b$ is given by the following optimization problem.

$$
c_{b k}^{j}=\arg \max _{i=1, \ldots, N} \quad \hat{\mathbf{x}}_{b k}^{\mathrm{T}} \hat{\mathbf{c}}_{i} .
$$

In problem (14), we use notation $c_{b k}^{j}$ to denote that analog beam $\mathbf{c}_{j}$ is the best solution for $k$ th user of BS $b$. If multiple users, $k \in \mathcal{K}_{b}$, have a same solution (say, $\mathbf{c}_{j}$ ), we can randomly chose any user and assign it with beam $\mathbf{c}_{j}$. Then for the remaining users, the analog beams are selected by creating a subset of codebook $\mathcal{C}$ by removing all assigned beams, and then solve problem (14) on the subset of codebook $\mathcal{C}$. These steps are repeated until $N^{\mathrm{RF}}$ number of analog precoders are selected from codebook $\mathcal{C}$.

\section{2) Beam sweeping}

Beam sweeping is one of the important features of 5G-NR in mmWave communications [4]. Various search schemes such as exhaustive search or iterative (or hierarchical) search has been studied in literature [18] to determine suitable direction 


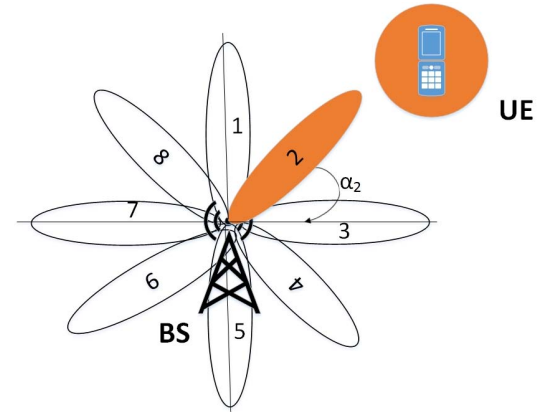

Fig. 1: User's position based analog beam design. Angle $\alpha_{2}$ is the angle of $2^{\text {nd }}$ beam with respect to an antenna array.

of transmission and reception. For problem (13), we can design analog beam by beam sweeping. In order to select analog beam, orthogonal pilots can be transmitted (in sequentially or parallel) by using all possible beams from the codebook $\mathcal{C}$. Then the best beam based on the generated receive power profile is assigned to user, see Fig. 2. This method is suitable for both LOS and non-LOS pathloss scenarios

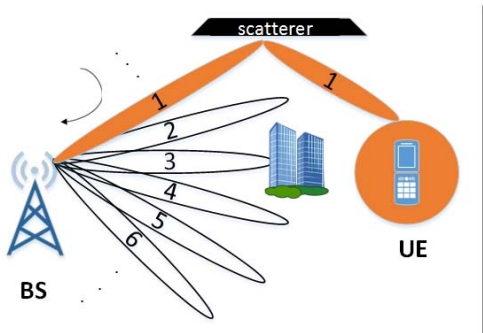

(a) beam sweeping

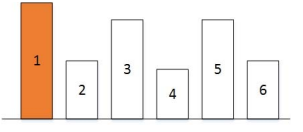

(b) receive power profile
Fig. 2: Beam sweeping illustration: analog beam 1 is associated for UE based on received power profile.

\section{B. Digital Precoder/Combiner Design}

In this section, for fixed analog precoder/combiner, we design digital precoder/combiner for problem (13) by exploiting a connection between WSRMax and WMMSE optimization problems. By following the approach in [19], we start by writing problem (13) equivalently as the following WMMSE minimization problem:

$$
\begin{aligned}
\operatorname{minimize} & \sum_{b \in \mathcal{B}_{\mathrm{DL}}} \sum_{k \in \mathcal{K}_{b}} \mu_{b k} \operatorname{Tr}\left(\mathbf{G}_{b k} \mathbf{E}_{b k}^{\mathrm{DL}}\right) \\
& +\sum_{n \in \mathcal{B}_{\mathrm{UL}}} \sum_{l \in \mathcal{K}_{n}} \bar{\mu}_{n l} \operatorname{Tr}\left(\overline{\mathbf{G}}_{n l} \mathbf{E}_{n l}^{\mathrm{UL}}\right)
\end{aligned}
$$

subject to constraints $(13 b)-(13 c)$,

with variables $\left\{\mathbf{V}_{b k}, \mathbf{W}_{b k}, \mathbf{G}_{b k}\right\}_{b \in \mathcal{B}_{\mathrm{DL}}, k \in \mathcal{K}_{\mathrm{b}}} \quad$ and $\left\{\overline{\mathbf{V}}_{n l}, \overline{\mathbf{W}}_{n l}, \overline{\mathbf{G}}_{n l}\right\}_{n \in \mathcal{B}_{U \mathrm{~L}}, l \in \mathcal{K}_{\mathrm{n}}}$. In expression (15), $\mathbf{G}_{b k}$ and $\overline{\mathbf{G}}_{n l}$ represents MMSE weight matrices and is given by [19]

$$
\mathbf{G}_{b k}=\left[\tilde{\mathbf{E}}_{b k}^{\mathrm{DL}}\right]^{-1}, \quad \overline{\mathbf{G}}_{n l}=\left[\tilde{\mathbf{E}}_{n l}^{\mathrm{UL}}\right]^{-1} .
$$

Problem (13) and (15) are equivalent in a sense that the optimal solution of (15) is also optimal for (13); we refer the interested reader to [19] for more detail.
Recall that in problem (15), the set of UL/DL beamformers $\left\{\mathbf{V}_{b k}, \overline{\mathbf{V}}_{n l}\right\}$, combiners $\left\{\mathbf{W}_{b k}, \overline{\mathbf{W}}_{n l}\right\}$, and the MMSE weight matrices $\left\{\mathbf{G}_{b k}, \overline{\mathbf{G}}_{n l}\right\}$ are optimization variables. Problem (15) is not jointly convex in these three set of variables. However, it is convex in each set of optimization variables $\left\{\mathbf{V}_{b k}, \overline{\mathbf{V}}_{n l}\right\}$, $\left\{\mathbf{W}_{b k}, \overline{\mathbf{W}}_{n l}\right\}$, or $\left\{\mathbf{G}_{b k}, \overline{\mathbf{G}}_{n l}\right\}$ (i.e., by keeping other two set of variables fixed). For fixed $\left\{\mathbf{V}_{b k}, \overline{\mathbf{V}}_{n l}\right\}$ and $\left\{\mathbf{G}_{b k}, \overline{\mathbf{G}}_{n l}\right\}$, the optimal combiners $\left\{\mathbf{W}_{b k}, \overline{\mathbf{W}}_{n l}\right\}$ are the LMMSE receivers and are given by the expressions (4) and (10), respectively. For fixed $\left\{\mathbf{V}_{b k}, \overline{\mathbf{V}}_{n l}\right\}$ and $\left\{\mathbf{W}_{b k}, \overline{\mathbf{W}}_{n l}\right\}$, the optimal solution for $\left\{\mathbf{G}_{b k}, \overline{\mathbf{G}}_{n l}\right\}$ is given by expression (16).

In the following section we derive an iterative algorithm to find precoders $\left\{\mathbf{V}_{b k}, \overline{\mathbf{V}}_{n l}\right\}$ for problem (15).

\section{1) Precoder optimization}

Problem (15) on variables $\left\{\mathbf{V}_{b k}, \overline{\mathbf{V}}_{n l}\right\}$ is a quadratic optimization problem [20] for fixed $\left\{\mathbf{W}_{b k}, \overline{\mathbf{W}}_{n l}\right\}$ and $\left\{\mathbf{G}_{b k}, \overline{\mathbf{G}}_{n l}\right\}$. Furthermore, this problem decouples across variables $\left\{\mathbf{V}_{b k}\right\}$ and $\left\{\overline{\mathbf{V}}_{n l}\right\}$, as the objective and constraints are separable on these two set of variables.

We first find solution for DL precoder $\left\{\mathbf{V}_{b k}\right\}$. Problem (15) on variable $\left\{\mathbf{V}_{b k}\right\}$ can be expressed as

$$
\begin{array}{ll}
\text { minimize } & \sum_{k \in \mathcal{K}_{\mathrm{b}}} \operatorname{Tr}\left(\mathbf{V}_{b k}^{\mathrm{H}} \Phi_{b} \mathbf{V}_{b k}\right)-2 \mathbb{R} \operatorname{Tr}\left(\mathbf{A}_{b k} \mathbf{V}_{b k}\right) \\
\text { subject to } & \sum_{k \in \mathcal{K}_{b}} \operatorname{Tr}\left(\mathbf{U}_{b} \mathbf{V}_{b k} \mathbf{V}_{b k}^{\mathrm{H}} \mathbf{U}_{b}^{\mathrm{H}}\right) \leq P_{b}^{\max }, b \in \mathcal{B}_{\mathrm{DL}},
\end{array}
$$

where $\mathbf{A}_{b k}=\mu_{b k} \mathbf{G}_{b k} \mathbf{W}_{b k}^{\mathrm{H}} \mathbf{H}_{b, b k} \mathbf{U}_{b}$ and $\boldsymbol{\Phi}_{b}$ is given by

$$
\begin{aligned}
& \boldsymbol{\Phi}_{b}=\sum_{p \in \mathcal{B}_{\mathrm{dl}}} \sum_{k \in \mathcal{K}_{p}} \mu_{p k} \mathbf{U}_{b}^{\mathrm{H}}\left(\mathbf{W}_{p k}^{\mathrm{H}} \mathbf{H}_{b, p k}\right)^{\mathrm{H}} \mathbf{G}_{p k}\left(\mathbf{W}_{p k}^{\mathrm{H}} \mathbf{H}_{b, p k}\right) \mathbf{U}_{b} \\
& +\sum_{n \in \mathcal{B}_{\mathrm{ul}}} \sum_{l \in \mathcal{K}_{n}} \bar{\mu}_{n l} \mathbf{U}_{b}^{\mathrm{H}}\left(\overline{\mathbf{W}}_{n l}^{\mathrm{H}} \overline{\mathbf{U}}_{n}^{\mathrm{H}} \tilde{\mathbf{H}}_{b, n}\right)^{\mathrm{H}} \overline{\mathbf{G}}_{n l}\left(\overline{\mathbf{W}}_{n l}^{\mathrm{H}} \overline{\mathbf{U}}_{n}^{\mathrm{H}} \tilde{\mathbf{H}}_{b, n}\right) \mathbf{U}_{b} .
\end{aligned}
$$

For the quadratic optimization problem (17), we can easily find the optimal solution by solving Karush-Kuhn-Tucker (KKT) conditions, and the solution is given by

$$
\mathbf{V}_{b k}=\left(\boldsymbol{\Phi}_{b}+\lambda_{b k} \mathbf{U}_{b}^{\mathrm{H}} \mathbf{U}_{b}\right)^{-1} \mathbf{A}_{b k}^{\mathrm{H}},
$$

where $\lambda_{b k}$ can be found using bisection search to satisfy power constraint $\sum_{k \in \mathcal{K}_{b}} \operatorname{Tr}\left(\mathbf{U}_{b} \mathbf{V}_{b k} \mathbf{V}_{b k}^{\mathrm{H}} \mathbf{U}_{b}^{\mathrm{H}}\right) \leq P_{b}^{\max }$.

We now solve problem (15) for UL precoder $\left\{\overline{\mathbf{V}}_{n l}\right\}$, and it can be expressed as

$$
\begin{array}{ll}
\operatorname{minimize} & \operatorname{Tr}\left(\overline{\mathbf{V}}_{n l}^{\mathrm{H}} \bar{\Phi}_{n l} \overline{\mathbf{V}}_{n l}\right)-2 \mathbb{R} \operatorname{Tr}\left(\overline{\mathbf{A}}_{n l} \overline{\mathbf{V}}_{n l}\right) \\
\text { subject to } & \operatorname{Tr}\left(\overline{\mathbf{V}}_{n l} \overline{\mathbf{V}}_{n l}^{\mathrm{H}}\right) \leq \bar{P}_{n l}^{\max }, \quad n \in \mathcal{B}_{\mathrm{UL}}, l \in \mathcal{K}_{\mathrm{n}},
\end{array}
$$
with variable $\left\{\overline{\mathbf{V}}_{n l}\right\}_{n \in \mathcal{B}_{\mathrm{UL}}, l \in \mathcal{K}_{\mathrm{n}}}$, and where $\overline{\mathbf{A}}_{n l}=$ $\bar{\mu}_{n l} \overline{\mathbf{G}}_{n l} \overline{\mathbf{W}}_{n l}^{\mathrm{H}} \overline{\mathbf{U}}_{n}^{\mathrm{H}} \mathbf{H}_{n, n l}^{\mathrm{H}}$ and $\overline{\mathbf{\Phi}}_{n l}$ is given by

$$
\begin{gathered}
\bar{\Phi}_{n l}=\sum_{b \in \mathcal{B}_{u l}} \sum_{k \in \mathcal{K}_{b}} \bar{\mu}_{b k}\left(\overline{\mathbf{W}}_{b k}^{\mathrm{H}} \overline{\mathbf{U}}_{n}^{\mathrm{H}} \mathbf{H}_{b, n l}\right)^{\mathrm{H}} \overline{\mathbf{G}}_{b k}\left(\overline{\mathbf{W}}_{b k}^{\mathrm{H}} \overline{\mathbf{U}}_{n}^{\mathrm{H}} \mathbf{H}_{b, n l}\right) \\
+\sum_{b \in \mathcal{B}_{d l}} \sum_{k \in \mathcal{K}_{b}} \mu_{b k}\left(\mathbf{W}_{b k}^{\mathrm{H}} \overline{\mathbf{H}}_{n l, b k}\right)^{\mathrm{H}} \mathbf{G}_{b k}\left(\mathbf{W}_{b k}^{\mathrm{H}} \overline{\mathbf{H}}_{n l, b k}\right) .
\end{gathered}
$$

Problem (19) is also a quadratic optimization, and hence its solution by KKT conditions can be obtained as

$$
\overline{\mathbf{V}}_{n l}=\left(\overline{\mathbf{\Phi}}_{n l}+\lambda_{n l} \mathbf{I}\right)^{-1} \overline{\mathbf{A}}_{n l}^{\mathrm{H}},
$$

where $\lambda_{n l}$ can be found using bisection search to satisfy power constraints $\operatorname{Tr}\left(\overline{\mathbf{V}}_{n l} \overline{\mathbf{V}}_{n l}^{\mathrm{H}}\right) \leq \bar{P}_{n l}^{\max }$. 
Finally, in Algorithm 1 below we summarize the proposed iterative algorithm to solve problem (13).

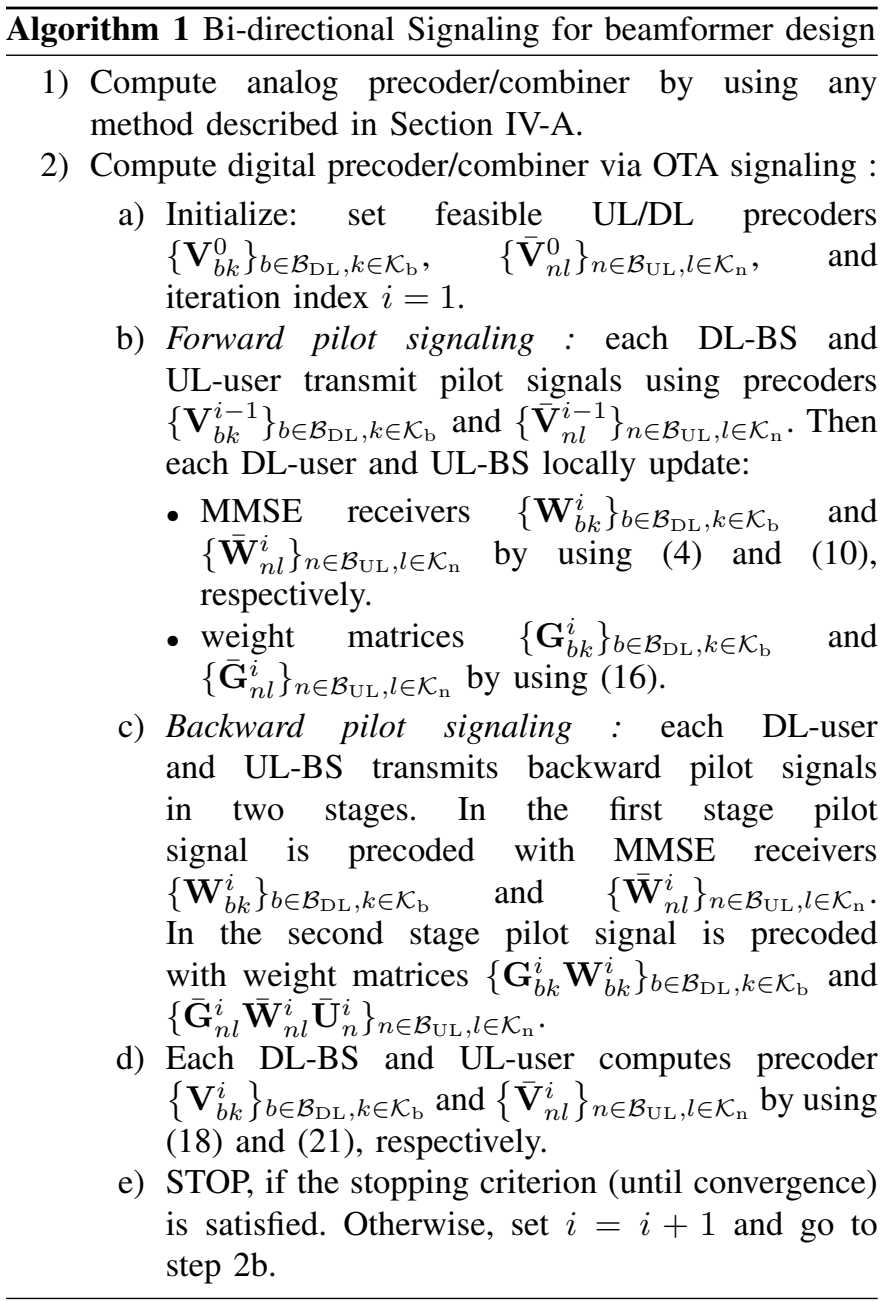

Algorithm 1 starts by computing analog precoder/combiner by using any one method discussed in Section IV-A. Then digital precoder/combiner is computed. We adopt OTA signaling approach in step 2 [8]. We assume orthogonal pilots for the forward and backward pilot signaling. These orthogonal pilots are precoded with either precoders, MMSE filters, or its scaled version. Hence, with the use of forward and backward pilot signaling we can easily extract all the required terms to compute precoder/combiner using (4), (10), (18), and (21) without a need of actual channel estimation. This strategy is essential for large scale MIMO systems.

\section{Simulation Results}

In this section, we illustrate the performance of the proposed Algorithm 1 using the setup as shown in Fig. 3. We consider $B=3$ cells (i.e., BSs), where two cells are in DL mode and one in UL mode. Each BS consist of $N=32$ antennas. We assume that 4 users are associated with each BS and each user is equipped with $M=2$ antennas. We assume $\mathrm{BS}$ and user equipment (UE) communicate via $d=1$ data stream.

A geometric path loss model with $L$ scatterring cluster is considered. Furthermore, we assume uniform linear array

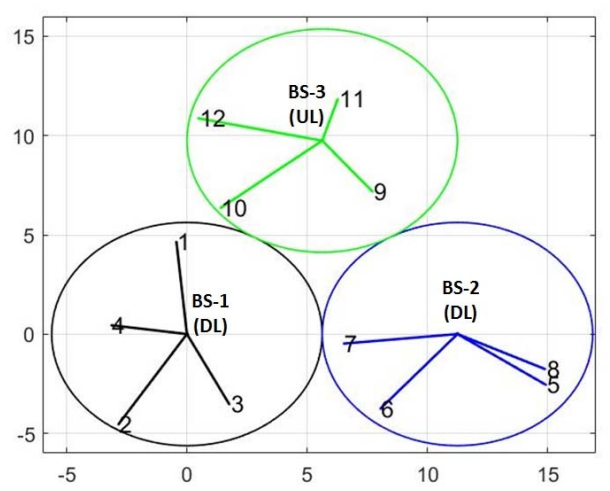

Fig. 3: UL/DL configuration of BSs and user distribution.

(ULA) antenna configuration [11]. Thus, a channel matrix between DL BS $b$ and $k$ th user of BS $b$ can be modeled as $\mathbf{H}_{b, b k}=\sqrt{\frac{M N}{L}}\left(\frac{d_{b, b k}}{d_{0}}\right)^{-\frac{\eta}{2}} \sum_{l=1}^{L} \alpha_{b, b k}^{l} \mathbf{a}_{\mathbf{r}}\left(\theta_{b, b k}^{l}\right) \mathbf{a}_{\mathbf{t}}\left(\phi_{b, b k}^{l}\right)^{\mathrm{H}}$, where $d_{b, b k}$ is the distance from BS $b$ to $k$ th user of BS $b, d_{0}$ is the far field reference distance, $\eta$ is the path loss exponent, and notation $\alpha_{b, b k}^{l} \in \mathcal{C N}(0,1)$ is the complex gain of $l$ th path. Array response vectors $\mathbf{a}_{\mathbf{r}}\left(\theta_{b, b k}^{l}\right)$ and $\mathbf{a}_{\mathbf{t}}\left(\phi_{b, b k}^{l}\right)$ are given by $\mathbf{a}_{\mathbf{r}}\left(\theta_{b, b k}^{l}\right)=1 / \sqrt{M}\left[1, e^{-j w \rho \cos \theta_{b, b k}^{l}}, \ldots, e^{-j w \rho(M-1) \cos \theta_{b, b k}^{l}}\right]^{\mathrm{T}}$ $\mathbf{a}_{\mathbf{t}}\left(\phi_{b, b k}^{l}\right)=1 / \sqrt{N}\left[1, e^{-j w \rho \cos \phi_{b, b k}^{l}}, \ldots, e^{-j w \rho(N-1) \cos \phi_{b, b k}^{l}}\right]^{\mathrm{T}}$, where $w=2 \pi / \lambda, \lambda$ is wavelength, and $\rho$ is antenna spacing. Similarly, BS-BS and UE-UE channels are modeled. In the simulation, we set $L=8, \rho=\lambda / 2,\left(\theta_{b, b k}^{l}\right) \in[0,2 \pi)$, and $\left(\phi_{b, b k}^{l}\right) \in[0,2 \pi)$. We generate analog codebook $\mathcal{C}$ by using the ULA antenna array response [11, Ch. 7].

We define signal-to-noise ratio (SNR) operating point at cell edge as $\operatorname{SNR}(R)=\left(R / d_{0}\right)^{-\eta} P_{b}^{\max } / \sigma^{2}$, where $R$ is the radius of the cell. We set $R$ fixed throughout the simulations such that $\operatorname{SNR}(R)=10 \mathrm{~dB}$ for $P_{b}^{\max } / \sigma^{2}=40 \mathrm{~dB}$. In the following simulations, we set $d_{0}=1, \eta=4$. Further, we assume equal priority weight for all users, i.e., $\left\{\mu_{b k}=1\right\}$ and $\left\{\bar{\mu}_{n l}=1\right\}$.

To illustrate the performance of Algorithm 1, we consider fully-digital MIMO system as a benchmark since it allows greater flexibility with more degree of freedom to implement efficient beamforming algorithm and provides better performance. Furthermore, we also consider alternating projection algorithm (APA) as described in [21] to get analog precoder/combiner, and design digital beamformers by using OTA training step 2 of Algorithm 1. We refer this method as APA-OTA.

Fig. 4 shows the average weighted sum-rate (WSR) versus SNR for different values of $N^{\mathrm{RF}}$ in LOS scenario. Each plot is averaged over 500 channel realizations. Results show that both position-based and beam sweeping for analog precoder/combiner achieve similar performance for various values of $N^{\mathrm{RF}}$. This is because in LOS scenario, same set of analog beams are optimal for both position-based and beam sweeping methods. Moreover, results show that both of our proposed methods outperform APA-OTA. 


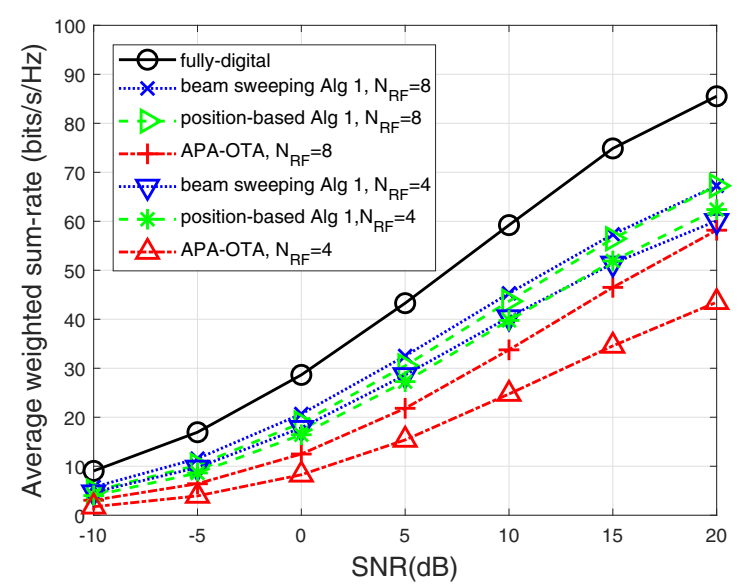

Fig. 4: LOS scenario: average WSR versus SNR

Fig. 5 shows the WSR versus SNR for different values of $N^{\mathrm{RF}}$ in non-LOS scenario. Results show that beam sweeping method in designing analog beams performs better than the position-based. Furthermore, results show that APA-OTA method slightly outperforms position-based method. However, it should be noted that CSI is required in APA-OTA method to design analog beamformer which is considerably challenging to obtain in large scale MIMO system.

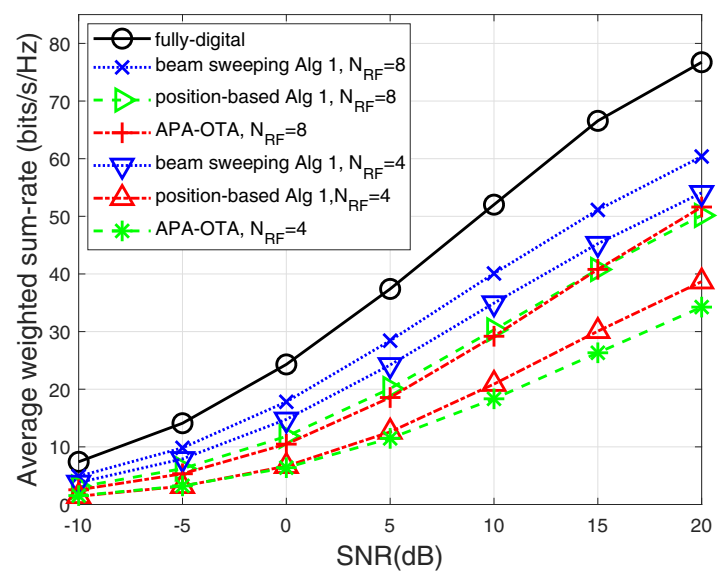

Fig. 5: Non-LOS scenario: average WSR versus SNR

\section{Vi. CONCLUSions}

We have considered the problem of weighted sum-rate maximization for hybrid architecture in millimeter wave communications with dynamic time division duplexing. We have proposed an over the air pilot-aided beamforming technique by exploiting the channel reciprocity. Proper selection of analog precoder/combiner not only suppresses large part of interference, but also ensures that less coordination is required for digital precoder/combiner design. Analog beamformer is designed for each data stream which maximizes its desired signal strength, ignoring possible interferences. Then digital beamformer is designed such that inter-stream interference is further mitigated, maximizing the weighted sum-rate of the network.

\section{REFERENCES}

[1] P. C. et al., "The evolution path of 4G networks: FDD or TDD?" IEEE Communications Magazine, vol. 44, no. 12, pp. 42-50, 2006.

[2] P. Omiyi, H. Haas, and G. Auer, "Analysis of TDD cellular interference mitigation using busy-bursts," IEEE Trans. Wireless Commun., vol. 6, no. 7, pp. 2721-2731, Jul. 2007.

[3] G. J. R. Povey and M. Nakagawa, "A review of time division duplex-CDMA techniques," in Proc. IEEE Int. Symp. Spread Spectrum Techniques and Applications, vol. 2, 1998, pp. 630-633.

[4] T. R. 38.802, "Study on new radio access technology physical layer aspects," 2017.

[5] Z. Shen, A. Khoryaev, E. Eriksson, and X. Pan, "Dynamic uplinkdownlink configuration and interference management in TD-LTE," IEEE Comm. Mag, vol. 50, no. 11, pp. 51-59, Nov. 2012.

[6] P. Jayasinghe, A. Tölli, J. Kaleva, and M. Latva-aho, "Bi-directional signaling for dynamic TDD with decentralized beamforming," in Proc. IEEE Int. Conf. on Commun Workshop., Jun. 2015, pp. 185-190.

[7] A. Tölli, J. Kaleva, G. Venkatraman, and D. Gesbert, "Joint UL/DL mode selection and transceiver design for dynamic TDD systems," in Proc. IEEE Int. Glob. Conf. on Sign and Inform. Proc., Dec 2016.

[8] P. Jayasinghe, A. Tölli, J. Kaleva, and M. Latva-aho, "Bi-directional beamformer training for dynamic TDD networks," IEEE Trans. on Sig. Proc, vol. 66, no. 23, pp. 6252-6267, Dec. 2018.

[9] D. Lee and J. So, "Adaptive feedback bits and power allocation for dynamic TDD systems," KICS J. Commun. Netw., vol. 21, no. 2, 2019.

[10] E. Cavalcante, G. Fodor, Y. Silva, and W. Cruz, "Bidirectional sumpower minimization beamforming in dynamic TDD MIMO networks," IEEE Trans. Veh. Technol., vol. 68, no. 10, pp. 9988-10 002, Oct. 2019.

[11] D. Tse and P. Viswanath, Fundamentals of wireless communication. Cambridge university press, 2005.

[12] T. S. Rappaport, S. Sun, R. Mayzus, H. Zhao, Y. Azar, K. Wang, G. N. Wong, J. K. Schulz, M. Samimi, and F. Gutierrez, "Millimeter wave mobile communications for $5 \mathrm{G}$ cellular: It will work!" IEEE access, vol. 1, pp. 335-349, 2013.

[13] F. Sohrabi and W. Yu, "Hybrid digital and analog beamforming design for large-scale antenna arrays," IEEE J. Select. Topics Signal Processing, vol. 10, no. 3, pp. 501-513, Apr. 2016.

[14] L. Liang, W. Xu, and X. Dong, "Low-complexity hybrid precoding in massive multiuser MIMO systems," IEEE Commun. Lett., vol. 3, no. 6, pp. 653-656, Dec. 2014.

[15] S. Joshi and M. Juntti, "RF-chain ADC resolution trade-off in MIMO hybrid architecture," in Proc. Annual Asilomar Conf. Signals, Syst., Comp., Oct. 2018, pp. 259-263.

[16] P. Komulainen, A. Tölli, and M. Juntti, "Effective CSI signaling and decentralized beam coordination in TDD multi-cell MIMO systems," IEEE Trans. Signal Processing, vol. 61, no. 9, pp. 2204-2218, 2013.

[17] A. F. Molisch, V. V. Ratnam, S. Han, Z. Li, S. L. H. Nguyen, L. Li, and K. Haneda, "Hybrid beamforming for massive MIMO: A survey," IEEE Commun. Mag., vol. 55, no. 9, pp. 134-141, 2017.

[18] M. Giordani, M. Mezzavilla, C. N. Barati, S. Rangan, and M. Zorzi, "Comparative analysis of initial access techniques in 5G mmwave cellular networks," in Proc. Conf. Inform. Sciences Syst. (CISS), Mar. 2016.

[19] S. S. Christensen, R. Agarwal, E. D. Carvalho, and J. M. Cioffi, "Weighted sum-rate maximization using weighted MMSE for MIMOBC beamforming design," IEEE Trans. Wireless Commun., vol. 7, 2008.

[20] S. Boyd and L. Vandenberghe, Convex optimization. Cambridge university press, 2004.

[21] J. Mo, A. Alkhateeb, S. Abu-Surra, and R. W. Heath, "Hybrid architectures with few-bit ADC receivers: Achievable rates and energyrate tradeoffs," IEEE Trans. Wireless Commun., vol. 16, Apr. 2017. 